\title{
DERIVING GENERAL RELATIVITY FROM STRING THEORY
}

\author{
NICK HUGGETT, UNIVERSITY OF ILLINOIS AT CHICAGO \\ AND \\ TIZIANA VISTARINI, RUTGERS UNIVERSITY
}

\section{INTRODUCTION}

The goal of this paper is to explain the significance of the conformal symmetry of string theory. Along the way we will introduce the basics of string theory in a streamlined fashion, drawing on familiar ideas from classical and quantum field theory. We will then explain how general relativity is a 'consequence' of string theory: not merely in the sense that it contains massless spin-2 particles - gravitons - but in the very strong sense that the coherent states of the graviton obey the Einstein field equations - gravitons truly form the gravitational field. This result follows from reimposing conformal symmetry in quantized string theory; so in the final section of the paper we sketch some more esoteric considerations justifying this assumption.

\section{The Formalism}

2.1. The Classical String. We will start with a classical relativistic string ${ }^{1}$, an object of one spatial dimension and one temporal dimension - it's best to think of it as a spacetime object from the get go. Let us suppose that it is 'closed', meaning that its ends are joined into a loop (figure 2.1 shows an open string - to close it, the timelike edges should be identified). Our string is free, subject to internal tension, but (for now) under the influence of no external forces, including gravity, so that it lives in Minkowski spacetime, with metric $\eta_{\mu \nu}$. Suppose that the points of the string come labelled with 'internal spacetime coordinates' $\tau$ and $\sigma$ (later $\sigma_{0}$ and $\sigma_{1}$ ); while 'external' or 'target' spacetime has coordinates $X^{\mu}$ $(\mu=0,1, \ldots, D-1)$. Then we can describe the string worldsheet in spacetime by assigning appropriate coordinates $\left(X^{0}, X^{1}, \ldots, X^{D-1}\right)$ to each internal point $(\sigma, \tau)$; formally there is a $D$-component vector field on the string. From the point of view of the string then, motion in target space amounts to changes in this field. This picture will be important as we progress, so bear it in mind.

So how do we expect this 2-dimensional object to behave? One's mind turns to Hooke's law, but that is uncongenial to relativity - Lorentz contraction should not change the tension in a string. What Hooke's law tells us more generally is that a string will minimize its length: again, not relativistically invariant, but close - the relativistic statement is that a string will minimize its spacetime area. Thus the simplest classical, relativistic string

\footnotetext{
${ }^{1}$ Here we draw heavily on several recent text-books, especially [Becker et al. (2006), Kiritsis (2011), Polchinski (2003), Zwiebach (2004)].
} 


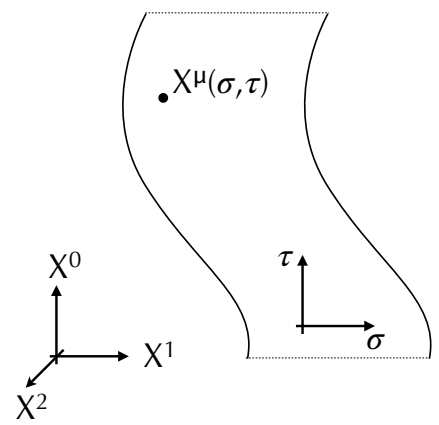

FiguRE 1. An open string in target space - if the timelike edges are identified then it becomes a closed string.

action is proportional to the invariant area $S=-T \int \mathrm{d} A$. Explicitly, $\mathrm{d} A=\sqrt{-g} \cdot \mathrm{d} X^{\mu} \mathrm{d} X^{\nu}$, or transforming into string coordinates, we obtain the famous Nabu-Goto action:

$$
S_{N G}=-T \int \mathrm{d} \sigma^{2} \sqrt{-\operatorname{det}\left(\eta_{\mu \nu} \frac{\partial X^{\mu}}{\partial \sigma^{\alpha}} \frac{\partial X^{\nu}}{\partial \sigma^{\beta}}\right)} .
$$

$T$ is the tension in the string (though you can't immediately see this from the form of the action); it makes clear that the string does not satisfy Hooke's law, because it is an invariant constant. The action also shows that all that matters is the total length of the string, not how parts might be stretched relative to one another - again un-Hooke-like behaviour. So, for one thing, the dynamics has no way of identifying parts of the string over time (the action has diffeomorphism symmetry with respect to the $\sigma \mathrm{s}$ ) - but the significance of this behaviour is far greater. ${ }^{2}$

The square root in $S_{N G}$ is awkward, but a formal trick leads to the equivalent sigma (or Polyakov) action:

$$
S_{\sigma}=-\frac{T}{2} \int \mathrm{d}^{2} \sigma \sqrt{-\gamma} \gamma^{\alpha \beta} \eta_{\mu \nu} \frac{\partial X^{\mu}}{\partial \sigma^{\alpha}} \frac{\partial X^{\nu}}{\partial \sigma^{\beta}} .
$$

The 'trick' involves introducing a second 'internal' metric, $\gamma_{\alpha \beta}$ on the string worldsheet to be carefully distinguished from the restriction of the spacetime metric to the string.

Now the un-Hooke-like behaviour of the string manifests itself in the fact that intervals with respect to the internal metric have no physical significance. The action appears to depend on how the string is stretched along its length - the derivatives are determined by the distance in external space separating points on the worldsheet separated by an infinitesimal distance in the string coordinates. But the behavior of the string that we have been stressing means that such infinitesimal distances have no physical significance,

\footnotetext{
${ }^{2}$ Quick aside: in fact one can give a Hooke's law treatment of the string, not in inertial coordinates, but in 'light cone' coordinates, in which one spatial coordinate is 'boosted to the speed of light'. Such coordinates are used in most text-books at some point.
} 
and so it should make no difference if any is rescaled by an arbitrary factor. In short, the action must be Weyl, or conformally invariant, and indeed it is: as can be readily checked, the sigma action is unchanged by $\gamma_{\alpha \beta} \rightarrow e^{\omega(\tau, \sigma)} \gamma_{\alpha \beta} \cdot{ }^{3}$

A couple of short notes. First, the action is conformally invariant with respect to the string metric, not the target space metric! For $\eta$ the relevant symmetry is Poincaré invariance. (The other symmetry of the action is diffeomorphism invariance with respect to both the $\sigma \mathrm{s}$ and $X \mathrm{~s}$.) Second, although we have been stressing the connection of conformal invariance to the un-Hooke-like behavior of a relativistic string, we did so mainly to illustrate how string theory is grounded in some very familiar physics. Conformal invariance will be crucial in what follows, but all we need is the straight-forward mathematical fact that $S_{\sigma}$ has that symmetry - not any story about why. For now we have the following: from the point of view of the string, string theory concerns a $D$-dimensional conformal field, living on a 2-dimensional spacetime (i.e., the worldsheet). That picture was central to the developments of the 'second string revolution' of the 1990s, and generally is the one that we will adopt.

The symmetries can be used to set the worldsheet metric flat:

$$
S_{\sigma}=\frac{T}{2} \int \mathrm{d}^{2} \sigma \dot{X}^{2}-X^{\prime 2}
$$

where the derivative are with respect to the worldsheet coordinates. ${ }^{4}$ The corresponding Hamiltonian is:

$$
H=\frac{T}{2} \int d \sigma \dot{X}^{2}+X^{\prime 2}
$$

and minimizing with respect to $X^{\mu}$ yields a wave equation,

$$
\ddot{X}^{\mu}-X^{\prime \prime \mu}=0 \text {. }
$$

The general solution for a closed string is (after a little more work in classical wave physics):

$$
X^{\mu}=X_{0}^{\mu}+\ell_{s}^{2} p^{\mu} \tau+i \frac{\ell_{s}}{\sqrt{2}} \sum_{n \neq 0} \frac{1}{n}\left(\alpha_{n}^{\mu} e^{-i 2 n(\tau-\sigma)}+\bar{\alpha}_{n}^{\mu} e^{-i 2 n(\tau+\sigma)}\right),
$$

where $\ell_{S}$, the 'characteristic string length', is determined by the tension: $\ell_{s}^{2}=1 / T$. This equation describes an initial position, linear momentum, and left- and right-moving vibrations - the $\alpha_{n}$ are the amplitudes of the modes of the string. Identifying the linear momentum as the zeroth mode of the string will be useful.

\footnotetext{
${ }^{3}$ More carefully, we have been talking about Weyl symmetry; 'conformal' transformations are strictly a sub-group of the diffeomorphisms, namely those whose only effect is to introduce a Weyl factor. This point is, for instance, important for understanding why conformal symmetry remains in (3), even though the Weyl symmetry has been gauge fixed.

${ }^{4}$ In the following the reader is especially referred to [Becker et al. (2006), §2.2-3]
} 


$$
\alpha_{0}^{\mu} \equiv \frac{\ell_{s}}{2} p^{\mu} \equiv \bar{\alpha}_{0}^{\mu}
$$

Substituting the mode expansion of $X^{\mu}$ into the Hamiltonian (4) gives

$$
H=\sum_{n=-\infty}^{\infty}\left(\alpha_{-n} \cdot \alpha_{n}+\tilde{\alpha}_{-n} \cdot \tilde{\alpha}_{n}\right)=0
$$

2.2. Immediate Consequences. Now, because of conformal symmetry, the variation of the action with respect to rescaling the metric must vanish:

$$
0=\frac{1}{\sqrt{-h}} \frac{\delta S_{\sigma}}{\delta h^{\alpha \beta}}=-2 T \pi\left(\partial_{\alpha} X \cdot \partial_{\beta} X+\frac{1}{2}\left(\begin{array}{cc}
-1 & 0 \\
0 & 1
\end{array}\right)_{\alpha \beta}\left(\dot{X}^{2}-X^{\prime 2}\right)\right) .
$$

The four equations given by the possible values of $\alpha$ and $\beta$ can be solved, and if the expansion for $X^{\mu}$ is inserted, entail that:

$$
\forall m \in \mathbb{Z} \quad 0=\frac{1}{2} \sum_{n=-\infty}^{\infty} \alpha_{m-n} \cdot \alpha_{n} \equiv L_{m} .
$$

The $L_{m}$ and $\tilde{L}_{m}$ are crucial objects in the formalism describing the string, the 'Virasoro generators', and the constraints (10) play a vital role in the theory. Physically speaking, from the worldsheet perspective, (9) gives $T_{\alpha \beta}$, the stress-energy tensor of the 2-dimensional stringy spacetime, and the Virasoro generators are its modes. Geometrically speaking, they are the generators of conformal transformations on the worldsheet.

As an example, consider the role of the constraints in determining the mass spectrum of the string. Observed at scales well above its characteristic length, intuitively a string will appear as a (spatially) point-like object - a particle - since its extension 'can't be seen'. Since the string appears as a particle, its linear four-momentum must satisfy the usual relation to its rest mass. Using (7):

$$
-M^{2}=p^{2}=\frac{2\left(\alpha_{0}^{2}+\tilde{\alpha}_{0}^{2}\right)}{\ell_{s}^{2}} .
$$

But the $m=0$ Virasoro constraint yields

$$
L_{0}=\frac{1}{2} \sum_{n=-\infty}^{\infty} \alpha_{-n} \cdot \alpha_{n}=0 \Rightarrow \frac{\alpha_{0}^{2}}{2}=-\sum_{n=1}^{\infty} \alpha_{-n} \cdot \alpha_{n},
$$

and similarly for $\tilde{L}_{0}$. So, using (8) 


$$
-M^{2}=\frac{4}{\ell_{s}^{2}} \sum_{n=1}^{\infty}\left(\alpha_{-n} \cdot \alpha_{n}+\tilde{\alpha}_{-n} \cdot \tilde{\alpha}_{n}\right)=\frac{4}{\ell_{s}^{2}} H .
$$

In other words, it follows from the constraint that the 'particle-mass' of a string depends on its vibrational modes - different vibrations give different 'particles'. Moreover, the Hamiltonian is proportional to the mass squared of an excited string, not (as might have been expected in relativity) the mass.

2.3. Quantization. In this paper we employ both canonical and path integral quantization: either way, $X^{\mu}$ is a field on a 2-dimensional Minkowski spacetime - the string worldsheet. Thus we start with equal-time commutation relations on the 'field':

$$
\left[X^{\mu}(\sigma), \Pi^{\nu}\left(\sigma^{\prime}\right)\right]=i \eta^{\mu \nu} \delta\left(\sigma-\sigma^{\prime}\right)
$$

which entails via 6 that

$$
\left[\alpha_{m}^{\mu}, \alpha_{n}^{\nu}\right]=m \eta^{\mu \nu} \delta_{m+n}
$$

Hence the quantized $\alpha$ s are raising and lowering operators, as one should expect. Our earlier analysis now shows that the quantized mass spectrum is discrete: it includes massless photons and gravitons, and importantly, for later work, a new masless scalar, the 'dilaton' (as well as negative mass tachyon modes). For suitable string tensions, the modes can reproduce the observed masses of meson families. However, the appropriate tension for quantum gravity is much greater, so observed particles are not theorized to be mode excitations of the string. (The mass spectrum of the standard model, is reproduced in a more complex way - relying, for instance, on compactified dimensions or D-branes.)

(15) tells us that for $m \neq 0$ the Virasoro generators can be obtained by simply replacing the $\alpha$ s in (10) with operators; while $L_{0}$ requires normal ordering. Omiting all details, the resulting commutation relations are found to be:

$$
\left[L_{m}, L_{n}\right]=(m-n) L_{m+n}+\frac{D}{12} m\left(m^{2}-1\right) \delta_{m+n, 0}
$$

the (classical) algebra of conformal generators, plus a 'central charge' term, which indicates a quantum 'anomaly', a breakdown of classical conformal invariance. Restoring the symmetry requires $D=26$ and leads to the infamous compactified dimensions of string theory. In what follows we explain another consequence of the anomaly.

\section{General Relativity from String Theory}

Consider the sigma-action, (2) but with a general Lorentzian metric:

$$
S_{\sigma}=-\frac{1}{\alpha^{\prime}} \int \mathrm{d}^{2} \sigma \sqrt{-\gamma} \gamma^{\alpha \beta} G_{\mu \nu} \partial_{\alpha} X^{\mu} \partial_{\beta} X^{\nu}
$$


$\alpha^{\prime}$ is (up to a factor) the reciprocal of the tension - in worldsheet perturbation theory, an expansion parameter. Otherwise, the only change is $\eta_{\mu \nu} \rightarrow G_{\mu \nu}$. At this point you may think that $G$ is free parameter, to be inserted by hand - that the 'background' metric is independent of what the strings do. But you would be wrong - $G$ has to satisfy the sourcefree Einstein field equations (a result going back to [Friedan (1980)]; and conjectures going back to the 1970s). String theory requires general relativity (to lowest order).

The proof runs as follows ([Callan et al. (1985)], see [Gasperini (2007)] for more detail):

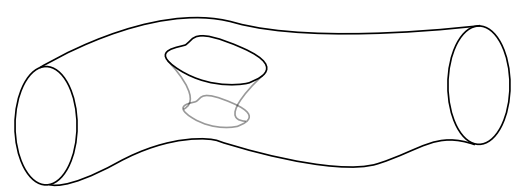

Figure 2. A string worldsheet with a 'hole' through it. This surface represents the first order correction to the closed string propagator - time is running left-to-right.

(1) The perturbative expansion of the interacting string propagator is a sum of tori with two legs - of increasing genus. Consider the first-order term in figure 3.

(2) Its contribution comes from a QFT defined by (17): from the point of view of the string, a 2-dimensional QFT - a 'non-linear sigma model' - in which $G_{\mu \nu}$ is a (varying) self-coupling for the $X^{\mu}$ field.

(3) This QFT has a well-studied perturbation theory, and is known to be renormalizable. (Here we have second perturbation expansion: one on the worldsheet, which is itself a term in the expansion of the string propagator.)

(4) Renormalization means counter-terms, which means running terms, with a length dependence. The renomalization group studies this dependence, describing the behavior in terms of $\beta$-functions.

(5) Friedan studied this renormalization group behavior, showing perturbatively that the $\beta$-function associated with $G_{\mu \nu}$ is - remarkably - given by $\beta_{G}=R_{\mu \nu}+O\left(\alpha^{\prime}\right)$, the Ricci tensor plus higher order corrections.

(6) Now, recall that we are talking about a field theory on a string worldsheet, so the length scale is with respect to the string metric, rather than the target space metric; but conformal invariance means that the theory can have no dependence on such a length! So the $\beta$-function vanishes, and by Friedan's work, $R_{\mu \nu}=0$, or $G_{\mu \nu}$ is 'Ricci flat' to lowest order. But that's equivalent to the vacuum Einstein field equations.

In short, the conformal invariance of string theory as a worldsheet QFT entails general relativity for target space. Of course, spacetime is not Ricci flat, but happily the result generalizes. To take a particularly salient example, suppose that strings live in a target space with a Yang-Mills field, a string theory with familiar matter. The action has the form: 


$$
S_{\sigma} \sim \frac{1}{\alpha^{\prime}} \int \mathrm{d}^{2} \sigma \mathrm{d} \theta G_{\mu \nu} \partial X^{\mu} \partial X^{\nu}+A_{\mu a}(X) \partial X^{\mu} j^{a}+\theta \psi^{i} \partial \psi^{i}
$$

This action is for a 'heterotic' string, featuring both conformal symmetry and supersymmetry between bose and fermi degrees of freedom: $\theta$ represents fermionic 'coordinates' in addition to the bosonic $\sigma$ s. $A$ is the background gauge field, and $\psi$ the fermions to which it couples ( $j$ their current). [Callan et al. (1985)] investigated this action, showing that to lowest order $G_{\mu \nu}$ is Ricci flat, and that the standard free Yang-Mills equations must be satisfied. Moreover, they also showed that at first order in $\alpha^{\prime}$ the $\beta$-function for the target metric has a term corresponding to the stress-energy of the Yang-Mills field.

$$
\beta_{G}=R_{\mu \nu}-\frac{\alpha^{\prime}}{2} \alpha^{\prime} \operatorname{tr}\left(F_{\mu \nu}^{2}\right)+O\left(\alpha^{\prime}\right) \text { terms for the coupling to } G_{\mu \nu}
$$

Thus, once again, worldsheet conformal symmetry, $\beta_{G}=0$, means that to order $\alpha^{\prime}$, the EFEs hold, even if when matter is present. However, we want to argue that these are more than formal results (we express what seems to be the view of string theorists). For the results do not simply show that strings tell matter and geometry backgrounds how to behave: they in fact describe how fields built from string excitations are related, so that the field equations are simply low energy descriptions of the string itself.

The reason is that 'background' fields do not represent new degrees of freedom in addition to those of string theory: they are not distinct primitive entities. Instead they represent the behavior of coherent states of string excitations: the quantum states, that is, which describe classical field behavior. Thus when one includes a general metric in the action, one has a quantum theory of perturbations around a coherent state corresponding to the given classical metric. The result shows that there is not a free choice of background fields, but that graviton and - in this case - Yang-Mills quanta coherent states must be related appropriately: by the EFEs.

More precisely, there are two claims involved in the view that background fields represent coherent states (both with evidence in their favor, [?, §3.6]): (i) that the string is an adequate 'theory of everything', in the sense that the string spectrum includes quanta for all desired background fields and (ii) that the terms in the action accurately capture the effective behavior of those coherent states. Then by (i) the $G_{\mu \nu}$ field is composed of stringy excitations, and by (ii) it satisfies the EFEs, making it the gravitational field, and the excitations gravitons. Thus, in the most literal sense, the general relativistic theory of spacetime is a low energy effective theory of strings.

If this is correct, then in a central sense, string theory is background independent: the metric arises from string interactions, rather than being stipulated a priori. Just like general relativity, many solutions are possible, but matter and gravity have to satisfy a mutual dynamics - except in string theory, there is no fundamental distinction between the two, a significant ontological unification.

What is left of the charge of background dependence? One might take the view that since the derivation starts with (2), which manifestly involves a Minkowski metric, at least that much geometry is 'background' (even if interpreted as an inner product on $X^{\mu}$ fields). 
It would still follow that the really interesting geometry of the theory is dynamical, and just an aspect of the same processes that constitute matter. But the situation is even better. For addressing the question of background independence, one should instead start with the more general action (17). The results above then show that the only possible metrics are Ricci flat, and so the only 'background' assumptions involves selecting one such metric. The idea that the geometry has to be 'put in by hand' hardly applies at all. .

\section{Towards a Philosophical Analysis of Conformal Symmetry}

The connections to the dimensionality of spacetime, and to the EFEs that we have sketched show that conformal symmetry is a key concept connecting string theory to phenomenological spacetime. (By 'phenomenological' we mean space and time as they are described by general relativity; and thus as they are envisioned in more-or-less direct experience, since the situations we normally experience fall within the domain of general relativity.) We claim that conformal symmetry should therefore be an important focus of philosophical attention in the study of string theory. [Huggett and Wüthrich (2013)] argues for the importance of philosophical analysis of the 'empirical significance' of such concepts in theories of quantum gravity: in short, such analyses promise to illuminate how (and even whether) aspects of spacetime can emerge from a theory which does not presuppose them at the fundamental level, in some sense. (The paper also explains the value of such analyses even for partial theories, such as bosonic string theory: the development of complete theories must be preceded by the development of suitable concepts, typically found in proto-form in incomplete theories.) This essay is a contribution to such an analysis, which we continue to develop in this section. The key question now is whether conformal symmetry is an independent postulate of string theory. We suggest not: the above results do not require extra assumptions, but are essential to string theory.

The story so far is that the classical string action is conformally invariant, but that this symmetry is broken by quantization. We have seen the effect of this 'conformal anomaly' in the central charge appearing in the Virasoro algebra, and in slightly more detail in the derivation of the EFEs, above. ${ }^{6}$

\footnotetext{
${ }^{5}$ Jeffrey Harvey has noted that even classical relativity requires some background, say in the form of asymptotic behaviour

${ }^{6}$ Now, the latter derivation was given in the context of renormalization, and so may appear to be a consequence of perturbation theory; one might assume that string theory does not require general relativity intrinsically, but only in order for a certain kind of approximation scheme to work. This would be a mistake (see e.g., [Nakahara (2003)]). The short story is this. Consider a schematic path integral for a quantum field:

$$
\int \mathcal{D} \varphi e^{i \int \mathbf{d} x \mathcal{L}[\varphi]}
$$

Manifestly, invariance of the Langrangian under a symmetry no longer suffices for a quantum symmetry: path integrals - hence amplitudes - will only be unchanged if the measure of the path integral is also invariant, and anomalies arise when it is not. Of course anomalies show up in the perturbative expansion of a path integral, but path integrals themselves are not inherently perturbative. Much more could be said on this subject, but not in this place.
} 
At this point one might wonder whether it is possible to abandon conformal symmetry. ${ }^{7}$ Thinking through the symmetries of the action (2), what this means is that Weyl transformations are no longer gauge symmetries, only diffeomorphisms are. Of course that would make a nonsense of the development of the string given earlier in this paper - indeed, it's simply false of the action classically! But the point just made is that the conformal anomaly means that the quantum system need not have the same symmetry. In this case of course different choices of conformal factor in the Weyl transformation of the internal metric, $\gamma_{\alpha \beta} \rightarrow e^{\omega(\tau, \sigma)} \gamma_{\alpha \beta}$, will be physically different: hence $\omega(\tau, \sigma)$ is a new physical degree of freedom over the worldsheet, in addition to, and prima facie rather alike, the $X^{\mu}$ s. However, $\omega \rightarrow \omega+\lambda$, a 'translation' in this new 'dimension', means a conformal transformation on the world sheet, since $e^{\omega+\lambda}=e^{\omega} e^{\lambda}$. Hence, if Weyl symmetry fails, so does translation symmetry in this new ' $\omega$-dimension'. For this reason the $\omega$ field cannot be just an additional target spacetime coordinate but requires a different interpretation, as a scalar background field. As a matter of fact, it has the form of a background dilaton field, $\Phi(X)$, the new string mode introduced earlier: more specifically (as we shall discuss) it is a linear dilaton field.

We shall explore the consequences of the failure of Weyl symmetry by investigating the dilaton - to summarize what we just said, its appearance is a direct consequence of violating the symmetry. On the one hand the dilaton allows one to relax some of the consequences of Weyl symmetry discussed so far. On the other, it will allow us to make good on our claim that Weyl symmetry is not an independent postulate of string theory, in the sense that it will in general signal a breakdown of perturbation theory.

To sketch the physics of the linear dilaton, we start with a new action including a background dilaton field $\Phi(X)$, as usual understood as representing physics around a coherent state (here of the scalar dilaton):

$$
\frac{1}{4 \pi \alpha^{\prime}} \int_{\Sigma} d^{2} \sigma \sqrt{-\gamma}\left[\left(\gamma^{a b} G_{\mu \nu}(X)+\alpha^{\prime} R \Phi(X)\right]\right.
$$

where $R$ is still the Ricci scalar, and $\alpha^{\prime}$ is still the expansion parameter of string perturbation theory (the reciprocal of the tension).

As we discussed above, formally (at least) this is the action for a two-dimensional interacting field theory. But the $X^{\mu}$ fields can be re-interpreted as target space coordinates; moreover at low energy it can be rewritten as an effective low energy action over spacetime:

$$
S_{X}=\frac{1}{2} \int d^{D} X \sqrt{-G} e^{-2 \Phi}\left[-\frac{2(D-26)}{3 \alpha^{\prime}}+R+4 \partial_{\mu} \Phi \partial^{\mu} \Phi+O\left(\alpha^{\prime}\right)\right] .^{8}
$$

Comparison of (21) and (22) shows that at low energy the perturbative expansion parameter $\alpha^{\prime}-$ (a function of) the string coupling - can be identified with $e^{2 \Phi(X)}$. This identification indicates the link between the presence of a linear dilaton and the applicability of string

\footnotetext{
${ }^{7}$ The remainder of the section draws heavily on [Polchinski (2003)] §3.4, 3.7 and 9.9.

${ }^{8}$ [Polchinski (2003), §3.7].
} 
perturbation theory: where the former diverges, the latter breaks down. Below we will indicate how controlling the divergences of the linear dilaton - hence the existence of string perturbation theory - leads back to Weyl invariance; for now, in order to explain some of the features of the linear dilaton, we will simply assume that result. More specifically, we assume that the $\beta$-functions for the action (22) vanish:

$$
\beta^{\Phi} \approx \frac{D-26}{6}+\alpha^{\prime}\left(\nabla^{2} \Phi+\nabla_{\omega} \Phi \nabla^{\omega} \Phi\right)+O\left(\alpha^{\prime 2}\right)=0
$$

and

$$
\beta^{G} \approx \alpha^{\prime}\left(R_{\mu \nu}+2 \nabla_{\mu} \nabla_{\nu} \Phi\right)+O\left(\alpha^{\prime 2}\right)=0 .
$$

The latter is a third example of how string theory entails the EFEs, in this case when gravity couples to a dilaton field. The simplest solution has a Minkowski target spacetime, $G_{\mu \nu}(X)=\eta_{\mu \nu}$, in which case

$$
R_{\mu \nu}=0 \quad \text { and } \quad \Phi(X)=V_{\mu} X^{\mu},
$$

where $V_{\mu}$ is a constant, so that the dilaton has a simple linear dependence on spacetime. But now (23) yields:

$$
D=26-6 \alpha^{\prime} V_{\mu} V^{\mu}
$$

Earlier we saw that in the absence of a dilaton field, Weyl symmetry requires that $D=26$ - the 'critical' dimension. Now we see that in the presence of a dilaton field $D$ can take on other values, less than or greater than 26 (depending on whether the gradient of the dilaton is spacelike or timelike). Thus the dilaton relaxes the consequences of Weyl invariance, as we mentioned earlier. It is interesting to notice that the dimension now appears to be a dynamical (though constant) feature of the theory, controlled by the (square of) the gradient of the dilaton $V_{\mu} V^{\mu}$.

Now that we have introduced the linear dilaton, we need to indicate why controlling dilaton divergences requires Weyl invariance. Why? Recall, when we attempted to break Weyl symmetry the conformal factor $\omega$ became a linear dilaton field, $\Phi(X)$. Moreover, the dilaton is related to the coupling, $\alpha^{\prime} \sim e^{2 \Phi(X)}$, so that its behavior signals the breakdown of perturbation theory. Thus violating Weyl symmetry requires that the dilaton divergences be controlled - but we shall now sketch how that itself requires Weyl invariance, so the attempt to violate the symmetry fails.

Consider a spacelike dilaton, $\Phi(X)=V_{1} X^{1}$. [Polchinski (2003), §9.9] notes that fixing divergencies at large $X^{1}$ can be achieved by introducing a 'tachyon profile', $\tau(x)$, into the action as a background field:

$$
S_{X}-\frac{1}{2} \int d^{D} X \sqrt{-g} e^{-2 \Phi}\left(g^{\mu \nu} \partial_{\mu} \tau_{\mu}(X) \partial_{\nu} \tau(X)-\frac{1}{\alpha^{\prime}} \tau^{2}(X)\right)
$$


where $S_{X}$ is the effective spacetime action (22). The equations of motion for the tachyon are then

$$
-\partial_{\mu} \partial^{\mu} \tau(X)+2 V^{\mu} \partial_{\mu} \tau(X)-\frac{4}{\alpha^{\prime}} \tau(X)=0
$$

whose solution is

$$
\tau(x)=\exp \left(q \cdot X^{1}\right) \quad \text { where } \quad q=\sqrt{\left(\frac{D-26}{6 \alpha^{\prime}}\right)} .
$$

Once again the target space action can be rewritten as an equivalent worldsheet action, this time of the form

$$
S_{\sigma}=\frac{1}{4 \pi \alpha^{\prime}} \int_{\Sigma} d^{2} \sigma \sqrt{-\gamma}\left[\left(\gamma^{a b} \eta_{\mu \nu} \partial_{a} X^{\mu} \partial_{b} X^{\nu}+\alpha^{\prime} R V_{1} X^{1}+\tau_{0} \exp \left(q_{1} \cdot x^{1}\right)\right],\right.
$$

where the dependence on $D$ of the action (31) is contained in

$$
q_{1}=\sqrt{\left(\frac{26-D}{6 \alpha^{\prime}}\right)}-\sqrt{\left(\frac{2-D}{6 \alpha^{\prime}}\right)} .
$$

For $D \leq 2, q_{1}$ is a positive quantity, so for $X^{1} \rightarrow+\infty$ the tachyon exponential gets large, suppressing this limit in the path integral, yielding an effective repulsive potential. But it is precisely for $X^{1} \rightarrow+\infty$ that the coupling

$$
\alpha^{\prime} \sim e^{2 \Phi(X)}=e^{2 \Phi(X)=V_{\mu} X^{\mu}}
$$

diverges. Hence the tachyon controls the theory for $D \leq 2$. For $D>2, q_{1}$ is complex, and the tachyon exponential is oscillatory, so the argument no longer holds. There's a dichotomy: either (as some have argued, [Polchinski (2003), 324]) some other mechanism is in play that prevents $X^{1} \rightarrow+\infty$, or the theory breaks down. But either way, or if $D \leq 2$, the only hope for a linear dilaton - and hence the only hope for the violation of Weyl symmetry - is a tachyon field, (28). But, according to [Polchinski (2003), 323], (28) is the condition for Weyl invariance of the dilaton, and hence Weyl invariance is unavoidable (unless the divergences can be controlled in another way).

\section{Conclusion}

In this paper we have attempted to sketch enough of string theory to sketch the significance of conformal symmetry. Of course this is a huge and complex subject, and we can barely claim to have scratched the surface. However, we have indicated its crucial consequences, and also its necessity. Thus we hope to have established our principal claim, that conformal symmetry of string theory deserves to be a focus of attention in the philosophical 
study of quantum gravity - itself one of the most pressing subjects within philosophy of physics.

\section{REFERENCES}

[Becker et al. (2006)] Katrin Becker, Melanie. Becker, and John H Schwarz, String theory and M-theory. Cambridge University Press, 2006.

[Callan et al. (1985)] Curtis G Callan, D Friedan, EJ Martinec, and MJ Perry, "Strings in background fields", Nuclear Physics B, 262(4):593-609, 1985.

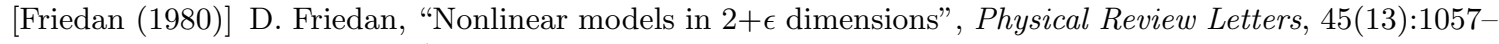
1060, 1980. doi: 10.1103/PhysRevLett.45.1057.

[Gasperini (2007)] Maurizio Gasperini, Elements of string cosmology. Cambridge University Press, 2007.

[Huggett and Wüthrich (2013)] Nick Huggett and Christian Wüthrich, "Emergent spacetime and empirical (in)coherence", Studies in History and Philosophy of Science Part B: Studies in History and Philosophy of Modern Physics, 44(3):276-285, 2013.

[Kiritsis (2011)] Elias Kiritsis, String theory in a nutshell. Princeton University Press, 2011.

[Nakahara (2003)] Mikio Nakahara, Geometry, topology and physics. CRC Press, 2003.

[Polchinski (2003)] Joseph Gerard Polchinski, String theory. Cambridge University Press, 2003.

[Zwiebach (2004)] Barton Zwiebach, A first course in string theory. Cambridge University Press, 2004. 